\title{
Bornological structures in the context of $L$-fuzzy sets
}

\author{
Aleksandrs Šostak ${ }^{1,2}$ Ingrīda Uljane ${ }^{1,2}$ \\ ${ }^{1}$ Department of Mathematics, University of Latvia, Zellu street, 8, Riga LV-1002, Latvia \\ ${ }^{2}$ Institute of Mathematics and Informatics, University of Latvia, Raina bulv., 29, Riga LV-1459, Latvia
}

\begin{abstract}
In order to apply the concept of boundedness, so crucial in the theory of metric spaces, to the case of a general topological space $\mathrm{Hu}$ Sze-Tsen introduced the notions of a bornological space and of boundedness for a mapping of bornological spaces. In this work we discuss two alternative approaches how the concepts of bornology and boundedness can be extended to the case of fuzzy sets and many-valued structures.
\end{abstract}

Keywords: Bornology, $L$-fuzzy bornology, $L$ valued bornology, bounded mapping, fuzzy metric, $D$-bounded set, $B$-bounded set.

\section{Introduction and motivation}

In order to apply the conception of boundness, so crucial in the theory of metric spaces, to the case of a general topological space $\mathrm{Hu}$ Sze-Tsen introduced the notions of a bornology and of a bornological space [14]:

Definition 1.1 [14] Given a set $X$ a bornology on it is a family $\mathcal{B} \subseteq 2^{X}$ of subsets of $X$ such that

(1B) $\forall x \in X \Longrightarrow\{x\} \in \mathcal{B}$;

(2B) if $U \subseteq V \subseteq X$ and $V \in \mathcal{B}$, then $U \in \mathcal{B}$;

(3B) if $U, V \subset X \quad U, V \in \mathcal{B}$ then $U \cup V \in \mathcal{B}$.

The pair $(X, \mathcal{B})$ is called a bornological space and the sets belonging to $\mathcal{B}$ are viewed as bounded in this space.

Thus actually a bornology on a set $X$ is an ideal in the powerset $2^{X}$ containing all finite sets. Important examples of bornologies spaces $(X, \mathcal{B})$ are: a topological space and its relatively compact subsets;

a metric space and its bounded subsets (that is sets with finite diameter);

a uniform space and its totally bounded subsets.

Definition 1.2 [14] Given bornological spaces $\left(X, \mathcal{B}_{X}\right)$ and $\left(Y, \mathcal{B}_{Y}\right)$ a mapping $f:\left(X, \mathcal{B}_{X}\right) \rightarrow\left(Y, \mathcal{B}_{Y}\right)$ is called bounded if the image $f(A)$ of every set $A \in \mathcal{B}_{X}$ belongs to $\mathcal{B}_{Y}$.

Further the theory of bornological spaces was developed by different authors, see e.g. [15] and the fundamental monograph by H. Hogle-Nled [12].
Aiming to develop an appropriate concept of bornology in the context of fuzzy sets and fuzzy structures we have to make a choice between different possible ways how it can be done. As a pattern of possible ways how this choice can be done we see the three well developed approaches to extension of the concept of topology to the context of fuzzy sets and fuzzy structures. Conceptionally generalizing these approaches to the case of a mathematical structure of a sufficiently general nature, we describe them as follows:

(FC) To consider a crisp analogue of a classical mathematical structure but to use families of fuzzy sets instead of families of ordinary sets. We call this approach a fuzzy-crisp approach. As a typical example of this approach one can think of Chang-Goguen fuzzy topological spaces [4], $[6],[7]$.

(CF) To consider fuzzy analogous of classical mathematical structures in case when the structure itself is fuzzy, but acts on families of crisp sets. Let us call it a crisp-fuzzy approach. As a typical example of this approach we have in mind Höhle-Ying's [13], [35] [36] definition of a fuzzy topology. Ying uses the name of a fuzzifying topology in this case.

(FF) Finally one can consider fuzzy analogues of classical mathematical structures when both the structure itself is fuzzy, and it acts on families of fuzzy sets. An example of this approach, which we refer to as a fuzzy-fuzzy approach is the concept of an $L$-fuzzy topology first defined in [26], [18] and later generalized in [27], [28], [19] [20] as the concept of an (LM)-fuzzy topology.

Although the last, that is fuzzy-fuzzy, approach is the most general one, our experience indicates that each one of these approaches has its own subject, field of research and applications as well as its own value, and therefore it makes sense, at least at the first stage of research, to develop the theory in the realms of each one of these approach separately.

In this work we briefly consider the theories of bornologies in the context of "Fuzzy Mathematics" thus far developed within the the first two approaches. The first one, fuzzy-crisp approach, is discussed in Section 3; its fundamentals were published in [1] and at present further research is being done in 
this direction. The second, fuzzy-crisp approach, is considered in Section 4. Its idea and basic concepts were announced at several conferences, see e.g. [31], [32], [33], [34], and it fundamentals are developed in in our paper [29]. In the last, Section 5, we consider the bornological structure of a fuzzy metric space.

The restricted volume of this paper did not allow us to include the detailed proofs here. The extended exposition of the problems studied in this work with detailed proofs can be found in $[1,29,30]$.

\section{Prerequisites: The context of our work}

\subsection{Lattices}

In our work $L$ denotes a fixed complete lattice $(L, \leq$ $, \wedge, \vee)$, that is a lattice in which the suprema (joins) and infima (meets) for all subfamilies $K \subseteq L$ exist.

In particular, the top $1_{L}$ and the bottom $0_{L}$ elements in $L$ exist and $0_{L} \neq 1_{L}$. We use notation $\vee$ and $\wedge$ to denote respectively, infima and suprema of finite families of elements of the lattice leaving notation $\bigwedge$ and $\bigvee$ for the case when these families are arbitrary.

In some cases we will additionally request the lattice $L$ to be completely distributive. Actually we will use not the original definition of complete distributivity, see e.g [5, Definition I-2-8], but its characterization found by G.N. Raney [23]. Namely, given a complete lattice $L$ and $\beta, \alpha \in L$ following [23], see also [5, Excercise IV-3-31], we introduce the so called "wedge below" relation $\triangleleft$ on $L$ as follows: ${ }^{1}$

$\beta \triangleleft \alpha$ if and only if when $K \subseteq L$ and $\alpha \leq \bigvee K$

then there there exists $\gamma \in K$ such that $\beta \leq \gamma$.

As shown by G.N. Raney [23] a lattice $L$ is completely distributive if and only if relation $\triangleleft$ has the approximation property, that is

$$
\alpha=\bigvee\{\beta \in L \mid \beta \triangleleft \alpha\}
$$

for each $\alpha \in L$. Moreover, relation $\triangleleft$ has the following important properties (see [5, 23]):

$(\triangleleft 1) \beta \triangleleft \alpha$ implies $\beta \leq \alpha$;

$(\triangleleft 2) \gamma \leq \beta \triangleleft \alpha \leq \delta$ implies $\gamma \triangleleft \delta$;

$(\triangleleft 3)$ if $\beta \triangleleft \alpha$ then there exists $\gamma \in L$ such that $\beta \triangleleft \gamma \triangleleft \alpha$

\section{2. cl-monoids}

To develop the theory in a sufficiently general, as we see it, context we present the basic definitions in the case when the lattice $L$ is equipped with a further binary operation $*: L \times L \rightarrow L$.

Definition 2.1 [25] Operation $*: L \times L \rightarrow L$ will be called a conjunction, or a t-norm if

\footnotetext{
${ }^{1}$ The authors are grateful to prof. M.A. De Prada Vicente for drawing their attention to the concept of a wedge below relation which found itself to be a very useful tool for this research.
}

(1t) $*$ is commutative: $\alpha * \beta=\beta * \alpha$ for all $\alpha, \beta \in L$;

$(2 \mathrm{t}) *$ is associative: $(\alpha * \beta) * \gamma=\alpha *(\beta * \gamma)$ for all $\alpha, \beta, \gamma \in L$

(3t) $\alpha * 1_{L}=\alpha, \quad \alpha * 0_{L}=0_{L}$ for all $\alpha \in L$.

Following Birkhoff [3], a complete lattice endowed with a conjunction, that is the tuple $(L, \leq, \wedge, \vee, *)$ will be called a cl-monoid. Operation * in a clmonoid is called lower semi-continuous if

(4t) * distributes over arbitrary joins:

$$
\begin{aligned}
& \alpha *\left(\bigvee_{i \in I} \beta_{i}\right)=\bigvee_{i \in I}\left(\alpha * \beta_{i}\right) \\
& \text { for every } \alpha \in L \text { and for all }\left\{\beta_{i} \mid i \in I\right\} \subseteq L .
\end{aligned}
$$

Following e.g. [16] we say that the t-norm * has no zero divisors, if

(5t) $\alpha * \beta \neq 0_{L}$ unless $\alpha \neq 0_{L}$ and $\beta \neq 0_{L}$.

Remark 2.2 In case $L=[0,1]$ is the closed unit interval, then the definition of a $t$-norm (in a slightly different form) for the first time appeared in [21]. Later it was thoroughly studied and applied in [25] and in a recently published monograph [16]. We extend the concept of a $t$-norm, which is very popular among the people working in the fields of "Fuzzy Mathematics", and use this term also in case when the unit interval $[0,1]$ is replaced by an arbitrary complete lattice $L$.

Example 2.3 Among the most important examples of $t$-norms $*$ are (see e.g. [16]):

- $*=\wedge$. In this case cl-monoid $(L, \leq, \wedge, \vee, *)$ just reduces to the underlying lattice $L$.

- Let $L=[0,1]$ and let $\alpha * \beta:=\alpha \cdot \beta$ be the product. Then we come to the so called product t-norm.

- Let $L=[0,1]$ and $\alpha * \beta=\min (\alpha+\beta, 1)$. Then $*$ is the well-known Łukasiewicz $t$-norm.

In the first two examples the $t$-norms do not have zero divisors, while the Łukasiewicz $t$-norm obviously has zero divisors.

From the definitions it is easy to verify the following well known

Proposition 2.4 If $L=(L, \leq, \wedge, \vee, *)$ is a clmonoid, then

$$
\alpha * \beta \leq \alpha \wedge \beta \text { for any } \alpha, \beta \in L
$$

\section{Bornological structures on families of $L$-fuzzy sets}

\subsection{Basic definitions}

Definition 3.1 An L-fuzzy bornology ${ }^{2}$ on a set $X$ is a family $\mathcal{B} \subseteq L^{X}$ such that

\footnotetext{
${ }^{2}$ In [1] the term $L$-bornology was used at this place. Now we change to $L$-fuzzy bornology in order to make a clear distinction between this kind of bornology-type structures and $L$-valued bornologies considered in Section 4
} 
(FB1) $\bigvee\{B \mid B \in \mathcal{B}\}=1_{X}$;

(FB2) $B \in \mathcal{B}, C \in L^{X}, C \leq B \Longrightarrow C \in \mathcal{B}$;

(FB3) $B_{1}, B_{2} \in \mathcal{B} \Longrightarrow B_{1} \vee B_{2} \in \mathcal{B}$.

The pair $(X, \mathcal{B})$ is called an $L$-fuzzy bornological space and $L$-sets $B \in \mathcal{B}$ are called bounded in this space.

An $L$-fuzzy bornology $\mathcal{B} \subseteq L^{X}$ will be called $a$ strict $L$-fuzzy bornology if it satisfies the following stronger version of the first axiom:

$\left(\mathrm{FB}^{\prime}\right) \mathbf{1}_{\{x\}} \in \mathcal{B} \forall x \in X$.

Remark 3.2 Obviously $\left(\mathrm{FB}^{\prime}\right) \Longrightarrow(\mathrm{FB} 1)$. If $1_{L} \in$ $L$ is isolated, that is $1_{L} \neq \sup M$ whenever $1_{L} \notin$ $M \subset L$ then $\left(\mathrm{FB}^{\prime}\right) \Longleftrightarrow(\mathrm{FB} 1)$. For example $\left(\mathrm{FB}^{\prime}\right) \Longleftrightarrow(\mathrm{FB} 1)$ in case $L=\mathbf{2}$ is the two-point lattice $\{0,1\}$. On the other hand generally (for example in case $L=[0,1])(\mathrm{FB} 1) \not\left(\mathrm{FB}^{\prime}\right)$.

Remark 3.3 Note that conditions (FB2) and (FB3) say that an $L$-fuzzy bornology on a set $X$ is just an ideal in the lattice $L^{X}$ while condition (FB1) is specific for this ideal.

Definition 3.4 Given two $L$-fuzzy bornological spaces $\left(X, \mathcal{B}_{X}\right)$ and $\left(Y, \mathcal{B}_{Y}\right)$ a mapping $f: X \rightarrow Y$ is called bounded if $f(B) \in \mathcal{B}_{Y}$ whenever $B \in \mathcal{B}_{X}$

Obviously if mappings $f:\left(X, \mathcal{B}_{X}\right) \rightarrow\left(Y, \mathcal{B}_{Y}\right)$ and $g:\left(Y, \mathcal{B}_{Y}\right) \rightarrow\left(Z, \mathcal{B}_{Z}\right)$ are bounded, then their composition $g \circ f:\left(X, \mathcal{B}_{X}\right) \rightarrow\left(Z, \mathcal{B}_{Z}\right)$ is bounded, too. Besides the identity mapping $i d_{X}:\left(X, \mathcal{B}_{X}\right) \rightarrow$ $\left(X, \mathcal{B}_{X}\right)$ is bounded. Hence $L$-fuzzy bornological spaces and bounded mappings between them form a category which will be denoted $L$-BOR and called the category of L-fuzzy bornological spaces.

In case $L=2$ is a two-point lattice the concept of a 2-fuzzy bornological space is obviously equivalent to the classical concept of a bornological space [12] and the category 2-BOR is actually the category of bornological spaces and bounded mappings.

\subsection{Lattice of $L$-fuzzy bornologies on a set}

Let a lattice $L$ and a set $X$ be fixed and let $\mathfrak{B}(X, L)$ be the family of all $L$-fuzzy bornologies on $X$. We introduce a partial order $\preceq$ on $\mathfrak{B}(X, L)$ by setting

$$
\mathcal{B}_{1} \preceq \mathcal{B}_{2} \Longleftrightarrow \mathcal{B}_{2} \subseteq \mathcal{B}_{1}, \mathcal{B}_{1}, \mathcal{B}_{2} \in \mathfrak{B}(X, L) .
$$

In this case we say that $L$-fuzzy bornology $\mathcal{B}_{2}$ is stronger than $L$-fuzzy bornology $\mathcal{B}_{1}$. Obviously, $(\mathfrak{B}(X, L), \preceq)$ is a partially ordered set. One can easily see that its bottom ( the weakest) element is given by $\mathcal{B}_{\perp}=L^{X}$.

To describe the top, i.e. the strongest element of $(\mathfrak{B}(X, L), \preceq)$ we introduce the following notations.

Given a set $A \subseteq X$ and a mapping $\lambda: A \rightarrow L_{0}$, where $L_{0}=L \backslash\{0\}$ let

$$
\operatorname{Pt}(A, \lambda)=\bigvee\left\{x^{\lambda(x)} \mid x \in A\right\} .
$$

In other words $\lambda$ is a mapping, assigning to each point $x \in A$ the value $\lambda(x) \in L_{0}$, thus creating a fuzzy point $x^{\lambda(x)}$. Further, let

$$
\operatorname{Pt}(A)=\left\{\operatorname{Pt}(A, \lambda) \mid \lambda \in\left(L_{0} \backslash\{1\}\right)^{A}\right\}
$$

and

$$
\operatorname{Pt}_{1}(A)=\left\{\operatorname{Pt}(A, \lambda) \mid \lambda \in L_{0}^{A}\right\} .
$$

If $1_{L}$ is isolated in $L$, then

$$
\mathcal{B}_{\top}:=\left\{\operatorname{Pt}_{1}(A)|A \subseteq X,| A \mid<\aleph_{0}\right\}
$$

is the strongest $L$-fuzzy bornology, as well as the strongest strict $L$-fuzzy bornology, on $X$. In case $1_{L}$ is not isolated in $L$, then $\mathcal{B}_{\top}$ is still the strongest strict $L$-fuzzy bornology on $X$, while the strongest $L$-fuzzy bornology is given by

$$
\mathcal{B}_{\top}:=\left\{\operatorname{Pt}(A)|A \subseteq X,| A \mid<\aleph_{0}\right\} .
$$

Theorem 3.5 ( cf Theorem 4.1 in [1]) Partially ordered set $(\mathfrak{B}(X, L), \preceq)$ is a complete infinitely distributive lattice. Its top and bottom elements are respectively $\mathfrak{B}_{\top}$ and $\mathfrak{B}_{\perp}$. Given a family $\mathfrak{B}_{0}=\left\{\mathcal{B}_{i} \mid i \in I\right\}$ its supremum is $\bigcap_{i \in I} \mathcal{B}_{i}$ and its infimum can be obtained as the family $<\mathcal{D}>$ of all finite joins of L-sets from the family

$$
\mathcal{D}=\bigcup\left\{B_{i} \mid B_{i} \in \mathcal{B}_{i}, i \in I\right\} \subseteq L^{X} .
$$

\subsection{Category $L$-BOR}

Given an $L$-fuzzy bornological space $\left(X, \mathcal{B}_{X}\right)$, a set $Y$ and a mapping $f:\left(X, \mathcal{B}_{X}\right) \rightarrow Y$ we define the final $L$-fuzzy bornological structure on $Y$ as follows.

Let $Y^{\prime}=f(X) \subseteq Y$. Further, let

$$
\mathcal{C}=\left\{f\left(B_{X}\right) \mid B_{X} \in \mathcal{B}_{X}\right\}
$$

Given $C \in \mathcal{C}$ we define

$$
\tilde{C}=\left\{\begin{aligned}
C(y) & \text { if } y \in Y^{\prime} \\
0_{L} & \text { if } y \notin Y^{\prime}
\end{aligned}\right.
$$

Further, let

$$
\begin{gathered}
\mathcal{B}_{Y}=\{\tilde{C} \mid C \in \mathcal{C}\} \cup\{\tilde{C} \vee D \mid C \in \mathcal{C}, D \in \operatorname{Pt}(A), \\
\left.A \subseteq Y \backslash Y^{\prime},|A|<\aleph_{0}\right\} .
\end{gathered}
$$

In other words, $\mathcal{B}_{Y}$ is defined as the family of all $L$-sets $E: L \rightarrow Y$ which are either of the form $\tilde{C}$ for some $C \in \mathcal{C}$ or are obtained as a join $\tilde{C} \vee D$ for some $C \in \mathcal{C}$ and some $L$-set $D:\left(Y \backslash Y^{\prime}\right) \rightarrow L$ with a finite support.

One can show that $\mathcal{B}_{Y}$ is an $L$-fuzzy bornology on $Y$, and moreover it is the strongest one for which the mapping $f:\left(X, \mathcal{B}_{X}\right) \rightarrow\left(Y, \mathcal{B}_{Y}\right)$ is bounded. Thus $\mathcal{B}_{Y}$ is the final $L$-fuzzy bornology for $f:\left(X, \mathcal{B}_{X}\right) \rightarrow$ $Y$. To obtain the final strict $L$-fuzzy bornology, we obviously have to use $\mathrm{Pt}_{1}(A)$ instead of $\operatorname{Pt}(A)$.

This result can be extended to the case of a family of mappings: 
Theorem 3.6 (cf Theorem 5.1 in [1])

Let $\left(X_{i}, \mathcal{B}_{i}\right), i \in I$, be a family of L-fuzzy bornological spaces and let $Y$ be a set. Given a sink, that is a family of mappings,

$$
\left\{f_{i}:\left(X_{i}, \mathcal{B}_{i}\right) \rightarrow Y \quad \mid i \in I\right\},
$$

there exists a unique final lift

$$
\left\{f_{i}:\left(X_{i}, \mathcal{B}_{i}\right) \rightarrow\left(Y, \mathcal{B}_{Y}\right) \mid i \in I\right\} .
$$

Corollary 3.7 Co-products exist in the category $L$ BOR.

Let now $f: X \rightarrow\left(Y, \mathcal{B}_{Y}\right)$. The weakest $L$ fuzzy bornology $\mathcal{B}_{X}$ on $X$ such that the mapping $f:\left(X, \mathcal{B}_{X}\right) \rightarrow\left(Y, \mathcal{B}_{Y}\right)$ is bounded can be described as

$$
\mathcal{B}_{X}=\left\{A \mid A \in L^{X}, \exists C \in \mathcal{C} \text { such that } A \leq C\right\},
$$

where $\mathcal{C}:=\left\{f^{-1}(B) \mid B \in \mathcal{B}_{Y}\right\}$.

This construction can be extended to the case of a family of mappings:

Theorem 3.8 Let $\left(Y_{i}, \mathcal{B}_{i}\right), i \in I$, be a family of $L$ fuzzy bornological spaces, and $X$ be a set. Given a source, that is a family of mappings,

$$
\left\{f_{i}: X \rightarrow\left(Y_{i}, \mathcal{B}_{i}\right) \mid i \in I\right\},
$$

there exists a unique initial lift

$$
\left\{f_{i}:\left(X_{i}, \mathcal{B}_{i}\right) \rightarrow\left(Y, \mathcal{B}_{Y}\right) \mid i \in I\right\} .
$$

Corollary 3.9 Products exist in the category LBOR

From Theorems 3.6 and 3.8 follows:

Theorem 3.10 The category of L-fuzzy bornological spaces and bounded mappings is topological [2] over the category SET of sets with respect to the forgetful functor $\mathfrak{F}: L$-BOR $\rightarrow$ SET .

\section{4. $L$-valued bornologies on powersets of sets}

\subsection{Basic definitions}

Let $(L, \leq, \wedge, \vee, *)$ be a cl-monoid and $X$ be a set.

Definition 4.1 An L-valued bornology on a set $X$ is a mapping $\mathcal{B}: 2^{X} \rightarrow L$ such that

(LB1) $\forall x \in X \quad \mathcal{B}(\{x\})=1$;

(LB2) If $U \subseteq V \subseteq X$ then $\mathcal{B}(V) \leq \mathcal{B}(U)$;

(LB3) $\forall U, V \subseteq X \quad \mathcal{B}(U \cup V) \geq \mathcal{B}(U) * \mathcal{B}(V)$.

The pair $(X, \mathcal{B})$ is called an L-valued bornological space and the value $\mathcal{B}(A)$ is interpreted as the degree of boundedness of a set $A$ in the space $(X, \mathcal{B})$.

In case if we want to emphasize the specific role of the $t$-norm in the cl-monoid $(L, \leq, \wedge, \vee, *)$ we use the term $(L, *)$-valued bornology in the above definition.
Note that in case $*=\wedge$, the second axiom (LB2) is redundant since it follows from the axiom (LB3) and hence $L$-valued bornology on a set $X$ can be defined as follows:

Definition 4.2 A mapping $\mathcal{B}: 2^{X} \rightarrow L$ where $(L, \leq, \wedge, \vee)$ is an infinely distributive lattice is an $(L, \wedge)$-valued bornology if and only if it satisfies conditions

(LB1) $\forall x \in X \quad \mathcal{B}(\{x\})=1$;

$\left(\mathrm{LB}^{\prime}\right) \forall U, V \subset X \quad \mathcal{B}(U \cup V)=\mathcal{B}(U) \wedge \mathcal{B}(V)$.

Moreover, from Proposition 2.4 it is clear that axiom (LB3') implies both of the axioms (LB2) and (LB3). Thus we have

Proposition 4.3 If $\mathcal{B}: 2^{X} \rightarrow L$ is an $(L, \wedge)$-valued bornology then it is an $(L, *)$-valued bornology for any $t$-norm in the lattice $L$.

Definition 4.4 A mapping $f:\left(X, \mathcal{B}_{X}\right) \rightarrow\left(Y, \mathcal{B}_{Y}\right)$ where $\left(X, \mathcal{B}_{X}\right),\left(Y, \mathcal{B}_{Y}\right)$ are $L$-valued bornological spaces is called bounded if $\mathcal{B}_{X}(A) \leq \mathcal{B}_{Y}(f(A))$ for every $A \in 2^{X}$.

Since obviously the composition of two bounded mappings of $L$-valued bornological spaces is bounded and the identity mapping is bounded, we conclude that $L$-valued bornological spaces and bounded mappings form a category which will be denoted $\mathbf{B O R}(L)$ and called the category of $L$ valued bornological spaces.

\subsection{Lattice of $L$-valued bornologies.}

Given a cl-monoid $(L, \leq, \wedge, \vee, *)$ and a set $X$ let $\mathfrak{B}(X, L, *)$ stand for the family of all $(L, *)$-valued bornologies on the set $X$. We introduce a directed "opposite point-wise" order relation $\preceq$ on $\mathfrak{B}(X, L, *)$, that is

$$
\mathcal{B}_{1} \preceq \mathcal{B}_{2} \Longleftrightarrow \mathcal{B}_{1}(A) \geq \mathcal{B}_{2}(A) \forall A \in 2^{X},
$$

and say in this case that $\mathcal{B}_{1}$ is coarser, or smaller than $\mathcal{B}_{2}$, and $\mathcal{B}_{2}$ is finer, or larger than $\mathcal{B}_{1}$. Obviously, $(\mathfrak{B}(X, L, *), \preceq)$ is a partially ordered set whose bottom element (that is the coarsest element) is defined by $\mathcal{B}_{\perp}(A)=1_{L}$ for all $A \in 2^{X}$, and whose top element (that is the finest element) is defined by

$$
\tilde{\mathcal{B}}_{T}(A)= \begin{cases}1_{L} & \text { if }|A|<\aleph_{0} \\ 0_{L} & \text { otherwise. }\end{cases}
$$

The tuple $(\mathfrak{B}(X, L, *), \preceq, \curlywedge, \curlyvee)$ becomes a complete lattice if the supremum $\curlyvee$ and the infimumum $\curlywedge$ in $(\mathfrak{B}(X, L, *), \preceq)$ are appropriately defined. We define them as follows.

Given a family

$$
\left\{\mathcal{B}_{i}: 2^{X} \rightarrow L \mid i \in I\right\}
$$

of $(L, *)$-valued bornologies, we define its supremum

$$
\curlyvee_{i \in I} \mathcal{B}_{i}=: \mathcal{B}^{0}: 2^{X} \rightarrow L
$$


by setting

$$
\mathcal{B}^{0}(A)=\bigwedge_{i \in I} \mathcal{B}_{i}(A)
$$

where $\bigwedge$ is the infimum in the lattice $L$. Thus we obtain an $(L, *)$-valued bornology $\curlyvee_{i \in I} \mathcal{B}_{i}$ on $X$ which is the supremum $\curlyvee$ of the family $\left\{\mathcal{B}_{i}: 2^{X} \rightarrow L \mid i \in\right.$ $I\}$ in the partially ordered set $(\mathfrak{B}(X, L, *), \preceq)$.

This already guarantees that the family $(\mathfrak{B}(X, L, *), \preceq, \curlyvee, \curlywedge) \quad$ of $\quad(L, *)$-valued bornologies is a complete lattice and the family $\mathfrak{B}(X, L)$ of $(L, \wedge)$-valued bornologies is its complete sublattice. Note however, that the point-wise supremum $\bigvee_{i}\left(\mathcal{B}_{i}(A)\right)$ of the family $\left\{\mathcal{B}_{i}: 2^{X} \rightarrow L \mid i \in I\right\}$ need not be an $(L, *)$-valued bornology (axiom (3LB) can be validated) and hence it need not be the infimum $\curlywedge$ of the family $\left\{\mathcal{B}_{i}: 2^{X} \rightarrow L \mid i \in I\right\}$ in the lattice $(\mathfrak{B}(X, L), \preceq, \curlywedge, \curlyvee)$. Therefore we define the infimum $\curlywedge$ of the family

$$
\left\{\mathcal{B}_{i}: 2^{X} \rightarrow L \mid i \in I\right\}
$$

by the equality

$$
\curlywedge_{i} \mathcal{B}_{i}(A)=\curlyvee\left\{\mathcal{B}_{j}(A) \mid \mathcal{B}_{j} \in \mathfrak{B}(X, L), B_{j} \leq \mathcal{B}_{i} \forall i \in I\right\} .
$$

An effective description of $\curlywedge$ is given in [29].

\subsection{Construction of $L$-valued bornologies from families of crisp bornologies}

Given an $(L, *)$-valued bornology $\mathcal{B}: 2^{X} \rightarrow L$ for every $\alpha \in L$ we define $\mathcal{B}_{\alpha}=\left\{A \in 2^{X} \mid \mathcal{B}(A) \geq \alpha\right\}$. In case $\alpha$ is an idempotent element of the $t$-norm $*$, the family $\mathcal{B}_{\alpha}$ is a crisp bornology on $X$. In particular, in case $*=\wedge$ all $\mathcal{B}_{\alpha}$ are crisp bornologies. Generally, however, $\mathcal{B}_{\alpha}$ may fail to be a crisp bornology due to validation of the $3^{\text {rd }}$ axiom.

Further, let $L$ be a completely distributive lattice and $*=\wedge$. Since in a completely distributive lattice every element is the supremum of a family of wedgebelow elements, it follows that the family $\left\{\mathcal{B}_{\alpha} \mid \alpha \in\right.$ $L\}$ is lower-semicontinuous from below in the sense that

$$
\mathcal{B}_{\alpha}=\bigcap\left\{\mathcal{B}_{\beta} \mid \beta \triangleleft \alpha, \beta \in L\right\} \text { for every } \alpha \in L,
$$

(in particular, $\mathcal{B}_{\top}=2^{X}$ as the intersection of the empty family)

and hence an $L$-valued bornology can be restored from its $\alpha$-level bornologies:

$$
\mathcal{B}(A)=\sup \left\{\beta \in L \mid A \in B_{\beta}(A)\right\} .
$$

We generalize the construction of restoration of an $L$-valued bornology from $\alpha$-level bornologies obtained by its decomposition and describe a construction of an $L$-valued bornology from an indexed family of crisp bornologies as follows.

Let $K$ be an approximative subset of $L$ (that is $\alpha=\sup \{\beta \in K \mid \beta \triangleleft \alpha\}$ for each $\alpha \in L)$ and let a non-increasing family of crisp bornologies on a set
$X$ be given: $\left\{\mathcal{C}_{\alpha} \mid \alpha \in K\right\}$ such that $\mathcal{C}_{0_{L}}=2^{X}$ For a set $A \subseteq X$ we define

$$
\mathcal{B}(A)=\lambda \text { where } \lambda:=\bigvee\left\{\alpha \in K \mid A \in \mathcal{C}_{\alpha}\right\} .
$$

Proposition 4.5 If the family $\left\{\mathcal{C}_{\alpha} \mid \alpha \in K\right\}$ is lower-semicontinuous, that is

$$
\mathcal{B}_{\alpha}=\bigcap\left\{\mathcal{B}_{\beta} \mid \beta \triangleleft \alpha, \beta \in K\right\} \text { for every } \alpha \in L,
$$

then the mapping $\mathcal{B}: 2^{X} \rightarrow L$ thus defined is an $L$-valued bornology and hence the pair $(X, \mathcal{B})$ is an L-valued bornological space. Moreover, $\mathcal{B}_{\alpha}=\mathcal{C}_{\alpha}$ for every $\alpha \in K$.

Since $\alpha \wedge \beta \geq \alpha * \beta$ axiom (LB3) is valid also for any $t$-norm $*: L \times L \rightarrow L$. we have the following

Corollary 4.6 The mapping $\mathcal{B}: 2^{X} \rightarrow L$ constructed in Proposition 4.5 is an $(L, *)$-valued bornology for any -norm $*$ on a completely distributive lattice $L$.

\subsection{Category of $L$-valued bornological spaces}

Let $(L, \leq, \wedge, \vee, *)$ be a cl-monoid.

Theorem 4.7 Every source

$$
\left\{f_{i}: X \rightarrow\left(Y_{i}, B_{i}\right) \mid i \in I\right\}
$$

has a unique initial lift

$$
\left\{f_{i}:\left(X, \mathcal{B}^{X}\right) \rightarrow\left(Y_{i}, \mathcal{B}_{i}\right) \mid i \in I\right\}
$$

in the category $\mathbf{B O R}(L)$ of L-valued bornological spaces.

The proof is given in [29]. The idea of the proof is as follows. Consider first the case of a single mapping $f: X \rightarrow\left(Y, \mathcal{B}_{Y}\right)$ and define $f^{-1}\left(\mathcal{B}_{Y}\right):=\mathcal{B}_{X}: 2^{X} \rightarrow$ $L$ by setting $\mathcal{B}_{X}(A)=\mathcal{B}_{Y}(f(A))$ for every $A \in 2^{X}$. Then $\mathcal{B}_{X}$ is an $(L, *)$-valued bornology, which is the smallest $(L, *)$-valued bornology in the lattice $(\mathfrak{B}(X, L, *), \preceq, \curlywedge, \curlyvee)$ of $(L, *)$-valued bornologies $\mathcal{B}$ on the set $X$ such that $f:(X, \mathcal{B}) \rightarrow\left(Y, \mathcal{B}_{Y}\right)$ is bounded. Coming now to the general case of a source $f_{i}: X \rightarrow\left(Y_{i}, \mathcal{B}_{i}\right), i \in I$ in the category $\operatorname{BOR}(L)$ we define the $L$-valued bornology on $X$ by setting $\mathcal{B}_{X}=\curlyvee_{i \in I} \mathcal{B}_{i}$, where $\curlyvee$ is the supremum in the lattice $(\mathfrak{B}(X, L, *), \preceq, \curlywedge, \curlyvee)$, that is

$$
\mathcal{B}_{X}(A)=\bigwedge_{i \in I}\left\{\mathcal{B}_{i}^{X}(A) \mid i \in I\right\}, \forall A \in 2^{X},
$$

and the $L$-valued bornology $\mathcal{B}_{i}^{X}=f_{i}^{-1}\left(\mathcal{B}_{i}\right)$ for each $f_{i}$ is defined as above. Then

$$
\left\{f_{i}:\left(X, \mathcal{B}^{X}\right) \rightarrow\left(Y_{i}, \mathcal{B}_{i}\right) \mid i \in I\right\}
$$

is the requested initial lift.

From Theorem 4.7 by duality principle we have 
Theorem 4.8 Every sink

$$
\left\{f_{i}:\left(X_{i}, \mathcal{B}_{i}\right) \rightarrow Y, i \in I\right\}
$$

has a unique final lift

$$
\left\{f_{i}:\left(X, \mathcal{B}_{i}\right) \rightarrow\left(Y, \mathcal{B}_{Y}\right), i \in I\right\}
$$

in the category $\mathbf{B O R}(L)$ of L-valued bornological spaces.

An efficient description of $\left(Y, \mathcal{B}_{Y}\right)$ is in [29].

From statements $4.7,4.8$ we obtain

Theorem 4.9 The category $\mathbf{B O R}(L)$ of L-valued bornological spaces and bounded mappings is topological over the category $\mathbf{S E T}$ with respect to the forgetful functor $\mathfrak{F}: \mathbf{B O R}(L) \rightarrow$ SET.

\section{5. $L$-valued bornologies induced by fuzzy metrics}

Since the bornology is the concept allowing to make a context for studying boundedness of mappings, and, in its turn, most clearly boundedness reveals itself in case of metric spaces, the first examples for $L$-valued bornologies also could be expected in situation when the underlying sets are equipped with, in this case fuzzy, metrics. So we start with a very brief introduction into the theory of fuzzy metrics.

\subsection{Fuzzy metrics}

Basing on the concept of a statistical metric [21], [25], I. Kramosil and J. Michalek in [17] introduced the notion of a fuzzy metric. Later A. George and P. Veeramani [8], [9] slightly modified the original concept of a fuzzy metric. This modification allows to produce more natural examples of fuzzy metrics which have nice properties and are important for applications. In particular, it allows to generate in a natural way a (crisp) Hausdorf topology on the underlying set. In this work we also base ourselves on George-Veeramani's notion of a fuzzy metric.

Definition $5.1[8,9]$ A fuzzy metric on a set $X$ is a pair $(M, \odot)$ such that $M: X \times X \times \mathbb{R}^{+} \rightarrow[0,1]$ is a fuzzy set, where $\mathbb{R}^{+}=(0,+\infty)$, and $\odot$ is a continuous t-norm satisfying the following conditions:

$(1 \mathrm{GV}) M(x, y, t)>0 \forall x, y \in X, \forall t \in(0, \infty)$;

$(2 \mathrm{GV}) M(x, y, t)=1$ if and only if $x=y$;

(3GV) $M(x, y, t)=M(y, x, t) \forall x, y \in X$, $\forall t \in(0, \infty)$;

$(4 \mathrm{GV}) M(x, z, t+s) \geq M(x, y, t) \odot M(y, z, s)$ $\forall x, y, z \in X \forall t, s \in(0, \infty)$;

$(5 \mathrm{GV}) M(x, y,-): \mathbb{R}^{+} \rightarrow[0,1]$ is continuous for all $x, y \in X$ as a function of $t$.

The triple $(X, M, \odot)$ is called a fuzzy metric space.

Definition 5.2 (see e.g. $[24,10,11,22]$ )

A fuzzy metric $M: X \times X \times(0, \infty) \rightarrow[0,1]$ is called strong or non-archimedian if the following stronger version of the axiom $(4 \mathrm{GV})$ is satisfied $\left(4^{s} \mathrm{GV}\right) M(x, z, t) \geq M(x, y, t) \odot M(y, z, t)$ for all $x, y, z \in X$ and for all $t>0$.

Remark 5.3 In case $\odot=\wedge$ the term a fuzzy ultrametric is also used when speaking about fuzzy metrics satisfying property $\left(4^{s} \mathrm{GV}\right)$, see e.g. [10, 11].

As different from the situation with topological structure of a fuzzy metric space studied, in particular, in $[8,9,11,10]$, we suggest to consider the corresponding bornological-type structure of this space as an essentially $L$-valued bornology on the powerset $2^{X}$. To do this in an appropriate way we start with discussing the concept of boundedness in fuzzy metric spaces. Unlike the situation in classical metric spaces where different definitions of boundedness are equivalent, in case of a fuzzy metric space fuzzy counterparts of these definitions may lead to different results. Below we discuss two approaches to the concept of boundedness in fuzzy metric spaces.

\subsection{Two types of boundedness in fuzzy metric spaces}

Let $(X, M, \odot)$ be a fuzzy metric space, $A \subseteq X$ and $t \in(0, \infty)$

Definition 5.4 A set $A$ is called locally B-bounded at a level $t$ or locally $B$-t-bounded for short, if there exist $\varepsilon \in(0,1)$ and $x_{0} \in X$ such that

$$
A \subset B_{t}\left(x_{0}, \varepsilon\right)=\left\{x \in X \mid M\left(x_{0}, x, t\right)>1-\varepsilon\right\} .
$$

$A$ set $A$ is called locally $B$-bounded if it is locally $B$-t-bounded for all levels $t \in(0, \infty)$.

Definition 5.5 A set A is called locally D-bounded at level $t$ or locally D-t-bounded for short, if $\operatorname{diam}_{t} A>0$, or, equivalently, if there exists $\varepsilon \in$ $(0,1)$ such that $\operatorname{diam}_{t} A>1-\varepsilon$ where the diameter $\operatorname{diam}_{t} A$ of set $X$ at a level $t$ is defined as

$$
\operatorname{diam}_{t} A=\inf \{M(x, y, t) \mid x, y \in A\} .
$$

$A$ set $A$ is called locally $D$-bounded if it is $D$-tbounded at all levels $t \in(0, \infty)$.

Note that the property of $F$-boundedness as it is defined in [8] is equivalent to our property of local $D$-t-boundedness for some $t \in(0, \infty)$.

Proposition 5.6 If a set $A$ is locally D-t-bounded, then it is also locally B-t-bounded.

Proposition 5.7 If the t-norm $\odot$ has no zerodivisors, and $A$ is locally B-t-bounded, then $A$ is also locally D-2t-bounded.

Corollary 5.8 Let $(M, \odot)$ be a strong fuzzy metric and let $\odot$ have no zero divisors. Then a set $A$ is locally B-t-bounded if and only if it is locally D-tbounded. 
Corollary 5.9 If $\odot$ has no zero divisors, then $A$ is locally B-bounded if and only if it is locally $D$ bounded.

Remark 5.10 Obviously, if a set $A$ is locally $B$ - $t$ bounded (resp. locally $D$-t-bounded) and $A^{\prime} \subset A$, then $A^{\prime}$ is also locally $B$-t-bounded (resp. $D$ - $t$ bounded). On the other hand the union $A=A_{1} \cup A_{2}$ of two locally $B$-t-bounded (resp. locally $D$ - $t$ bounded) $A_{1}$ and $A_{2}$ may fail to be locally $B$-tbounded (resp. locally $D$-t-bounded). A sufficient condition for preservation of local $B$-t-boundedness and local $D$ - $t$-boundedness is the request that the fuzzy metric $(M, \odot)$ is strong and the absence of zero-divisors for the $t$-norm $\odot$. Also one can prove that the union of two locally $B$-bounded (resp. locally $D$-bounded) sets is locally $B$-bounded (resp. locally $D$-bounded).

\section{3. $L$-valued bornologies induced by fuzzy metrics}

In this subsection $L=[0,1]$ and $*=\wedge$.

To construct an $L$-valued bornology $\mathcal{B}: 2^{X} \rightarrow L$ induced by a fuzzy metric $M: X \times X \times[0, \infty) \rightarrow$ $[0,1]$ we will use the construction of an $L$-valued bornology on a set $X$ from a family of usual bornologies $\mathcal{C}_{\alpha}$ on $X$ developed in Section 4 . The natural way to obtain such a family is to extract $\mathcal{C}_{\alpha}$ bornologies from locally $D$ - $t$-bounded or locally $B$ $t$-bounded families of sets in $X$. To realize this idea we have to establish natural relations between the set $(0, \infty)$, as the range of the parameter $t$, and the set $[0,1]$ as the original range of the parameter $\alpha$. Referring to Section 4.3 for this construction we may replace the closed interval $[0,1]$ by any approximating subset $K \subseteq[0,1]$. In our case it is natural to take $(0,1)$ in the role of $K$.

In order to find correspondence between the range $(0,+\infty)$ and the set $(0,1)=K$ one can take any strictly decreasing continuous bijection $\varphi$ : $(0, \infty) \rightarrow(0,1)$. As a typical example here we can take the hyperbola

$$
\varphi(t)=\frac{1}{1-t} \quad \forall t \in(0,+\infty)
$$

whose inverse $\psi:(0,1) \rightarrow(0, \infty)$ is defined by

$$
\psi(\alpha)=\frac{1-\alpha}{\alpha} \quad \forall \alpha \in(0,1) .
$$

Further, since the construction of an $L$-valued bornology presented here does not differ whether we start from locally $D$-t-bounded sets or locally $B$-t-bounded sets, we use just the term a locally $t$ bounded set.

Given a fuzzy metric space $(X, M, \odot)$ and $\alpha \in$ $(0,1)$ let $\mathcal{C}_{\alpha}$ stand for the family of finite unions of $\psi(\alpha)$-bounded subsets of the space $(X, M, \odot)$. One can easily notice that for every $\alpha$ the family of sets $C_{\alpha}$ is a crisp bornology on $X$ and the family $\left\{\mathcal{C}_{\alpha} \mid \alpha \in(0,1)\right\}$ is non-increasing. Hence we can apply the construction described in Subsection 4.3 to define an $L$-valued bornology $\mathcal{B}: 2^{X} \rightarrow[0,1]$ from the family $\left\{\mathcal{C}_{\alpha} \mid \alpha \in(0,1)\right\}$ of crips bornologies:

$$
\mathcal{B}(A)=\bigvee\left\{\alpha \in(0,1) \mid A \in \mathcal{C}_{\alpha}\right\} .
$$

Thus given a fuzzy metric space $(X, M, \odot)$ we construct an $L$-valued bornology $\mathcal{B}_{M}$. Since $\wedge \geq *$ for any $t$-norm $*, \mathcal{B}_{M}$ is an $(L, *)$-bornology also for any $t$-norm $*$, in particular, an $(L, \odot)$-bornology.

\section{References}

[1] M. Abel, A.Šostak, Towards the theory of $L$ bornological spaces, Iranian Journal of Fuzzy Systems, 8 No. 1, (2011) 19-28

[2] J. Adámek, H. Herrlich and G.E. Strecker, Abstract and Concrete Categories, John Wiley \& Sons, New York 1990.

[3] G. Birkhoff, Lattice Theory, AMS Providence, RI, 1995.

[4] C.L. Chang, Fuzzy topological spaces, J. Math. Anal. Appl., 24 (1968), 182-190.

[5] G. Gierz, K.H. Hofmann, K. Keimel, J.D. Lawson, M.W. Mislove, D.S. Scott, Continuous Lattices and Domains, Cambridge University Press, Cambridge, 2003.

[6] J.A. Goguen, L-fuzzy sets, J. Math. Anal. Appl., 18 (1967), 145-174.

[7] J.A. Goguen, The fuzzy Tychonoff theorem, J. Math. Anal. Appl., 43 (1973), 734-742.

[8] A. George, P. Veeramani, On some results in fuzzy metric spaces, Fuzzy Sets Syst., 64 (1994) 395-399.

[9] A. George, P. Veeramani, On some results of analysis for fuzzy metric spaces, Fuzzy Sets Syst., 90 (1997) 365-368.

[10] V. Gregori, J.-J. Miñana, S. Morillas, Some questions in fuzzy metric spaces, Fuzzy Sets Syst., 204 (2012) 71-85.

[11] V. Gregori, S. Morillas, A. Sapena On a class of completable fuzzy metric spaces, Fuzzy Sets Syst., 204 (2012) 71-85.

[12] H. Hogle-Nled Bornology and Functional Analysis, Math. Studies 26, North-Holland, Amsterdam, 1977.

[13] U. Höhle, Upper semicontinuous fuzzy sets and applications, J. Math. Anal. Appl., 78 (1980) 659-673.

[14] S.-T. Hu, Boundedness in a topological space, J. Math. Pures Appl., 78 (1949), 287-320.

[15] S.-T. Hu, Introduction to General Topology, Holden-Day, San-Francisko, 1966.

[16] E.P. Klement, R. Mesiar, E. Pap, Triangular norms, Kluwer Acad. Publ., 2000.

[17] I. Kramosil, J. Michalek, Fuzzy metrics and statistical metric spaces, Kybernetika 11 (1975), $336-344$.

[18] T. Kubiak, On Fuzzy Topologies, PhD Thesis, Adam Mickiewicz University, Poznań, Poland, 1985. 
[19] T. Kubiak, A.Šstak, A fuzzification of the category of $M$-valued L-topological spaces, Appl. Gen. Topology, 5(2) (2004) 137-154.

[20] T. Kubiak, A.Sostak, Foundations of the theory of $(L, M)$-fuzzy topological spaces, 30th Linz Seminar on Fuzzy Sets, Linz, Austria, February 3-8, 2009. Abstracts, pp. 70-73.

[21] K. Menger, Probabilistic geometry, Proc. N.A.S., 37 (1951), 226-229.

[22] D. Mihet, Fuzzy $\psi$-contractive mappings in non-archimedian fuzzy metric spaces, Fuzzy Sets and Syst., 159 (2008), 739-744.

[23] G.N. Raney, A subdirect-union representation for completely distibutive complete lattices, Proc. Amer. Math. Soc. 4 (1953), 518522.

[24] A. Sapena, A contribution to the study of fuzzy metric spaces, Appl. Gen. Topol., 2 (2001) 63-76.

[25] B. Schveizer, A. Sklar, Statisitcal metric spaces, Pacific J. Math. 10 (1960) 215-229.

[26] A. Šostak, On a fuzzy topological structure, Suppl. Rend. Circ. Matem. Palermo, Ser II 11 (1985), 125-186.

[27] A. Šostak, Two decades of fuzzy topology: Basic ideas, notions and results, Russian Math. Surveys, 44 (1989), 125-186

[28] A. Šostak, Basic structures of fuzzy topology, J. Math. Sci. 78 (1996), 662-701.

[29] A. Sostak, I. Uljane, Towards the theory on L-valued bornological spaces, Preprint submitted to Fuzzy Sets and Syustems (2013).

[30] I. Uljane, A. Šostak, Some remarks on $L$ fuzzy bornologies and L-valued bornologies, in preparation.

[31] I. Uljane, A. Šostak, On many-valued bornological structures, $15^{\text {th }}$ International Conference "Mathematical Modelling and Analysis"' Druskeninkai, Lithuania, May 2629, 2010, Astracts, p. 106.

[32] I. Uljane, A. Šostak, On bornological type structures in the context of L-fuzzy sets, 2010 Intern. Conf. on Topology and Appl., Nafpaktos, Greece. Abstracts, 226-229.

[33] I Uljane, On L-valued bornologies on powersets, The 2012 Ibero-American Conference on Topology and its Applications, Mexico Guanajuato, April 10-13, 2012, Abstracts, p. 43.

[34] I Uljane, Some remarks on L-valued bornologies in the context of fuzzy sets, International Conference on Topology and the Related Fields, September 23-25, 2012, Nanjing, China, Abstracts, p. 42.

[35] M.S. Ying, A new approach to fuzzy topology Part I, Fuzzy Sets and Syst., 39 (1991), 303321.

[36] M.S. Ying, A new approach to fuzzy topology, Part II, Fuzzy Sets and Syst., 47 (1992), 221-232.
[37] L.A. Zadeh, Fuzzy sets, Inform. and Control, 8 (1965), pp. 338-353. 